\title{
HOMEOPATIA APLICADA À REPRODUÇÃO ANIMAL
}

\author{
Luiz Carlos Cesar da Costa Filho \\ Vanessa Lopes Dias Queiroz ${ }^{1}$ \\ Mônica Filomena de Assis de Souza ${ }^{1}$ \\ Carmem Estefânia Serra Neto Zúccari ${ }^{1}$ \\ Eliane Vianna da Costa e Silva ${ }^{1}$
}

COSTA FILHO, L. C. C. da; QUEIROZ, V. L. D.; SOUZA, M. F. de A.; ZÚCCARI, C. E. S. N.; COSTA e SILVA, E. V. da. Homeopatia aplicada à reprodução animal. Arq. Ciênc. Vet.Zool. UNIPAR, Umuarama, v. 17, n. 1, p. 63-68, jan./mar. 2014.

RESUMO: A homeopatia é um sistema terapêutico que propõe abordagem clínica e terapêutica para o tratamento do indivíduo doente, desenvolvida por Christian Friedrich Samuel Hahnemann no final do século XVIII. No Brasil, a homeopatia foi introduzida por Benoit Mure, em 1840, tornando-se uma nova opção de tratamento. Como princípio básico tem-se a utilização de medicamentos dinamizados, ou seja, preparados a partir de substâncias animais, vegetais, minerais ou tecidos doentes. Existem duas leis que sustentam a homeopatia como parte da medicina. A primeira é a Lei dos Semelhantes - "Similia simili buscurentur"- (os semelhantes que se curem pelos semelhantes) e, a segunda, a Lei do Vitalismo. O uso da homeopatia na reprodução se faz importante na busca de melhorias da fertilidade animal, tanto no diz respeito ao tratamento de patologias quanto na eficiência reprodutiva. Contudo, novos estudos devem ser desenvolvidos, uma vez que há divergências nos resultados encontrados na literatura.

PALAVRAS CHAVE: Eficiência reprodutiva. Hahnemann. Terapia alternativa.

\section{HOMEOPATHY APPLIED TO ANIMAL REPRODUCTION}

\begin{abstract}
Homeopathy is a therapeutic system proposing both clinical and therapeutic approaches to the treatment of the patient, developed by Christian Friedrich Samuel Hahnemann in the late eighteenth century. In Brazil, homeopathy was introduced by Benoit Mure in 1840, becoming a new treatment option. The use of dynamized medication, or prepared from animal, vegetable or mineral substances, or from diseased tissue is at the core of homeopathy. There are two laws that support homeopathy as part of medicine. The first one is the Law of Similars - "Similia simili buscurentur" - (like cures like). The second law is the Law of Vitalism. The use of homeopathy in reproduction is important in the search for improvement in animal fertility, both regarding the treatment of diseases and as in reproductive efficiency. However, further studies still need to be developed, since there are differences in the results found in literature.
\end{abstract}

KEYWORDS: Alternative therapy; Reproductive efficiency; Hahnemann.

\section{HOMEOPATÍA APLICADA A LA REPRODUCCIÓN ANIMAL}

RESUMEN: La homeopatía es un sistema terapéutico que ofrece enfoque clínico y terapéutico para el tratamiento de la persona enferma, desarrollado por Christian Friedrich Samuel Hahnemann a finales del siglo XVIII. En Brasil, la homeopatía fue introducida por Benoit Mure en 1840, convirtiéndose en una nueva opción de tratamiento. Como principio básico se ha utilizado drogas energizadas, es decir, sustancias preparadas a partir de animales, vegetales, minerales o tejidos enfermos. Hay dos leyes que apoyan la homeopatía como parte de la medicina. La primera es la ley de los semejantes - "Similia simili buscurentur" - (los semejantes que se curen por los semejantes) y, la segunda, la Ley del Vitalismo. El uso de la homeopatía en la reproducción llega a ser importante en la búsqueda de la mejora de la fertilidad de los animales, tanto en lo que respecta al tratamiento de patologías como la eficiencia reproductiva. Sin embargo, nuevos estudios deben ser desarrollados, ya que hay divergencias en los resultados encontrados en la literatura.

PALABRAS CLAVE: Eficiencia reproductiva. Hahnemann. Terapia alternativa.

\section{Introdução}

A homeopatia é uma ciência da saúde que propõe abordagem clínica e terapêutica para o tratamento do indivíduo doente, desenvolvida por Christian Friedrich Samuel Hahnemann no final do século XVIII, quem primeiro a empregou em animais (KENT, 1993). Pode ser utilizada na terapêutica, assim como na prevenção de diversas afecções.

No Brasil, a homeopatia foi introduzida por Benoit Mure, em 1840, tornando-se uma nova opção de tratamento. Em 1979, fundou-se a Associação Médica Homeopática Brasileira (AMHB). Em 1980, a homeopatia tornou-se uma especialidade médica pelo Conselho Federal de Medicina (COSTA; ARAÚJO; FREITAS, 2010). Na medicina veterinária, a homeopatia passou a ser especialidade apenas em 1995, mediante a Resolução $n^{\circ}$ 625/95 do Conselho Federal de Medicina Veterinária e Zootecnia (BRASIL, 1995).

Como princípio básico tem-se a utilização de medicamentos dinamizados, ou seja, preparados a partir de substâncias animais, vegetais, minerais ou tecidos doentes. A matéria oriunda desses elementos impregna energeticamente as moléculas do álcool (ou açúcar) utilizado sem alterar sua forma química (ARENALES, 2002). 


\section{Desenvolvimento}

\section{As leis da homeopatia}

Existem duas leis que sustentam a homeopatia como parte da medicina. A primeira é a Lei dos Semelhantes - "Similia simili buscurentur"- (os semelhantes que se curem pelos semelhantes) e, a segunda, a Lei do Vitalismo (BENITES, 2006).

A Lei dos Semelhantes trata-se de uma lei natural que foi observada durante a evolução das moléstias quando, junto a elas, instala-se outro processo mórbido semelhante, esse interrompe ou cura o processo inicial. Um dos exemplos clássicos que Hahnemann utiliza no livro Organon é a não infecção de crianças por coqueluche quando elas já haviam sido acometidas por sarampo, pois as duas doenças guardam grande semelhança na natureza de sua febre e tosse (BENITES, 2006).

Hipócrates já citava a cura por similaridade, o qual denominava reação homeostática curativa, que encontra embasamento científico no estudo do "efeito rebote" ou "reação paradoxal" das drogas modernas. Por exemplo, drogas utilizadas no controle da hipertensão arterial podem provocar uma hipertensão arterial de rebote, como reação secundária do organismo ao estímulo primário.Porém, esse princípio é pouco aceito na farmacologia moderna, pois entra em conflito com o modelo terapêutico vigente que utiliza princípios terapêuticos dos contrários ou enantiopáticos (TEIXEIRA, 2006)

Sua segunda lei é explicada pela condição que rege e harmoniza o ser vivo, fenômeno imaterial que inexiste na substância mortal e que caracteriza a vida, na sua essência diferencia as matérias vivas das não vivas. Também em $O r$ ganon, Hahnemann descreve que no estado de saúde, a força vital de natureza espiritual, que dinamicamente anima o corpo material, reina com poder ilimitado e mantém todas as suas partes em atividade harmônica, nas suas sensações e funções (BENITES, 2006).

A partir das duas leis surgiram três princípios essenciais à prática da homeopatia: 1) Experimentação no indivíduo: sendo o principal pilar de sustentação da ciência, pois determina quais respostas dos diferentes organismos surgem ao estímulo de um mesmo medicamento; 2) Individualização: uma vez já conhecido os efeitos do medicamento é necessário conhecer o indivíduo, para decidir qual medicamento deve ser ministrado em um determinado momento; 3 ) Dinamização: é o princípio das pequenas doses ou infinitesimal (PIRES, 2005; BENITES, 2006)

Com a dinamização dos medicamentos são gerados dois outros processos: a diluição e a sucussão. A diluição possui três escalas diferentes, as hahnemannianas são centesimais $(\mathrm{C}$ ou $\mathrm{CH})$ e a cinquenta milesimal (LM) e a escala decimal (D, X ou $\mathrm{DH})$ que foram introduzidas na homeopatia por Hering. A sucussão corresponde à agitação do medicamento após cada diluição. A potência medicamentosa de cada composto é derivada de cada etapa do processo de dinamização (CESAR, 2003; BENITES, 2006).

Na prática a utilização de produtos homeopatizados baseia-se na comparação entre os sintomas apresentados pelo doente, repertorização, e os apresentados nas experimentações medicamentosas, permitindo assim a escolha correta em cada caso. Porém, apenas alguns experimentos foram conduzidos com animais e, embora a grande maioria da literatura médica seja humana, as adaptações devem ser feitas para tratar as demais espécies (PIRES, 2005; BENITES, 2006).

A escolha dos componentes homeopáticos do bionúcleo pode ser realizada com base no princípio hipocrático que determina o diagnóstico do enfermo por meio da sua totalidade de sintomas: comportamentais, gerais e locais (físicos), obtendo-se desta forma um medicamento, como princípio da terapêutica homeopática unicista (EIZAYAGA,1992).

A terapêutica utilizada nos princípios homeopáticos é a do estímulo do organismo para agir por si só, e não uma terapêutica que age no organismo, contrapondo-se a alguma ação dele ou mimetizando substância que ele mesmo produz. Neste sentido, o meio, e suas interações, que rodeiam o animal são importantes (AMARAL, 2002).

A homeopatia é uma ciência que individualiza o paciente, promovendo a integração entre seus sintomas físicos e suas características mentais. No entanto, ao aplicar-se a populações extensas, 100 animais ou mais, o tratamento pode ser realizado com segurança, pois o princípio denominado Genius epidemius, criado por Hahnemann, estará sendo aplicado (ARENALES, 2002).

A técnica é simples, pois todos os indivíduos que compõem o grupo, os animais a serem tratados, são considerados um único ser, como se um lote de bovinos fosse uma colmeia de abelhas, em que cada indivíduo, porém é, na realidade, a parte de um todo. Os sintomas são ordenados a partir da estatística de importância, selecionados homeopaticamente. Os sintomas mentais são considerados a partir da movimentação do grupo e não de indivíduos isolados. Dessa forma, o medicamento indicado é fornecido a todo o grupo. Essa técnica é importante na solução de epidemias humanas e hoje utilizada pelo Médico Veterinário homeopata como instrumento para curar e prevenir doenças no gado de corte (ARENALES, 2002; MITIDIERO, 2002; PIRES, 2005).

\section{Experimentação animal}

O tratamento homeopático de um rebanho apresenta algumas vantagens citadas por Souza (2002) e Lopes (2004) como equilíbrio animal (diminuição de estresse), facilidade de administração, inexistência de resíduos e ausência de contaminação do meio ambiente.

Trabalhando com terapia populacional, Macedo e Real (2008) testaram dois lotes de ovelhas Corriedale, em uma fazenda do Rio Grande do Sul, em que um grupo consumiu, durante o período de monta, composto homeopático e os animais controle, apenas sal mineral. A taxa de nascimento do grupo tratado foi $18,67 \%$ maior que o não tratado $(84,69 \%$ vs $66,01 \%)$, demonstrando ser vantajosa a prática homeopática populacional em ovinos.

Moncayo (2000) observou redução na contagem de células somáticas de vacas tratadas com homeopatia. Resultados satisfatórios no tratamento homeopático coletivo de mastite em vacas leiteiras, na Índia, foram relatados por Varshney e Naresh (2005), comparativamente ao uso de antibióticos. Por outro lado, Silva et al. (2011) não obtiveram diferença na contagem de células somáticas do leite para vacas Holandesas tratadas e não tratadas com homeopatia.

Martins et al. (2007) relataram redução da frequên- 
cia de mastite subclínica desde o primeiro mês de tratamento, mantendo-se em queda durante os dois meses subsequentes.

Trabalhando com bovinos machos castrados, zebuínos confinados, Ferreira (2005) observou que o grupo tratado com homeopatia, Calcarea carbonica 30CH (centesimal de Hahnemann) e Calcarea phosphorica $30 \mathrm{CH}$ na proporção de $1: 1$, veiculadas em carbonato de cálcio, apresentou um menor peso visceral ao abate e um vantajoso deslocamento dos depósitos de gordura interna para a região subcutânea. Nos dados de ganho de peso e consumo de matéria seca não foram encontradas diferenças significativas para os animais tratados ou não.

Soares Filho e Caetano (2000) relataram que bovinos de corte tratados com núcleo homeopático reduziram o consumo diário de matéria seca, prejudicando assim o seu desempenho, com diminuição no ganho de peso. Em ovinos, Chabel et al. (2009) também não encontraram evidências suficientes para atribuir ao complexo homeopático o resultado do ganho de peso de ovinos machos inteiros tratados com Convert $\mathrm{H}^{\circledR}$.

A prática homeopática contempla o bem-estar animal podendo, ser considerada uma alternativa aos medicamentos alopáticos, tendo menor custo e sua administração facilitada, pois pode ser diluído na água, ou misturado à ração ou sal mineral. Há uma diminuição das contenções e traumas, como pela aplicação de injeções e no manejo excessivo dos animais (ARENALES, 2002).

Em testes de laboratório com camundongos sob estresse por restrição alimentar, Teixeira et al. (2003) encontraram maior ganho de peso para fêmeas que receberam complexo homeopático quando comparadas com o grupo controle.

Chabel et al. (2009) identificaram uma redução significativa nos níveis de cortisol em ovinos submetidos à restrição alimentar quando os mesmos consumiram Convert $\mathrm{H}^{\circledR}$.

Quando o complexo homeopático Convert $\mathrm{H}^{\circledR}$ foi testado em camundongos machos, para verificar o efeito diante do estresse agudo, o produto não apresentou efeito significativo na diminuição do estresse dos roedores. O grupo que não foi submetido ao tratamento estressante apresentou efeito sobre o comportamento dos camundongos, o que reduziu a movimentação espontânea e aumentou o nível de ansiedade e de agressividade (LOPES et al., 2009).

Zorzatto et al. (2005) adicionaram $5 \mathrm{ml}$ de Conver$\mathrm{tH}^{\circledR}$ a $500 \mathrm{ml}$ de água durante dez gerações de uma colônia de camundongos SwissWebster (Mus musculus), enquanto a colônia controle não recebeu produto homeopatizado. $\mathrm{Na}$ avaliação dos parâmetros reprodutivos, o número médio de filhotes nascidos e de desmamados foi significativamente maior nas gerações da Colônia Convert $\mathrm{H}^{\circledR}$ quando comparado a não tratada. Porém, o número de partos não diferiu significativamente.

Benites (2006) descreve o caso clínico de uma vaca Holandesa com 10 anos de idade, tratada com um composto homeopático (Calcarea carbônica, Nuxvomica, Sepia e Sulphur) após apresentar prolapso uterino. A matriz foi inseminada por sete vezes sem sucesso. Iniciou-se o tratamento com o composto homeopático inicialmente com Sepia $6 \mathrm{CH}$ plus e, trinta dias após, com Sepia $30 \mathrm{CH}$ em dose única. A vaca foi submetida à superovulação resultando em três embriões viáveis e três prenhes. A vaca foi novamente insemi- nada, levando desta vez a gestação a termo.

No tratamento de ovariopatias císticas de bovinos de leite, Castilho et al. (2003) verificaram efeitos significativos do tratamento homeopático vs Ovsynch ${ }^{\circledR}(\mathrm{GnRH}$ e Prostaglandina) em vacas mestiças holandesas. O complexo homeopático utilizado foi composto por Thuya occidentalis $6 \mathrm{CH}$, Apis mellifera $6 \mathrm{CH}$ e Oophorinum $6 \mathrm{CH}$, escolhidos de acordo com o princípio da lei dos semelhantes. A homeopatia apresentou maior taxa de gestação que o tratamento hormonal após a primeira $(62,5 \%$ vs $22,22 \%)$ e segunda inseminação artificial $(87,5 \%$ vs 55,56\%), para homeopatia e Ovsynch ${ }^{\circledR}$,respectivamente.

Em um trabalho com búfalas e vacas, Kumar et al. (2004) induziram o estro em 50\% das fêmeas bubalinas e $68 \%$ das bovinas utilizando medicamentos homeopatizados (Calcarea phosphorica, Aletris farinosa, Pulsatilla, Aurummuriaticum natronatum, Sepia e Phosphorus, todos a uma potência de $30 \mathrm{CH})$.

Rajkumar et al. (2006), buscando minimizar e tratar o anestro em vacas, utilizaram o mesmo composto homeopático já testado por Kumar et al. (2004), e observaram a eficiência do medicamento, que induziu $100 \%$ de estro nas fêmeas, além de levar ao aumento na concentração de estradiol no grupo tratado. Os autores explicam que, talvez, este fato deva-se a presença de Pulsatilla e Aletris farinosa no composto, descritos como indutores de crescimento folicular.A utilização de Pulsatilla nigricans possibilitou uma redução no período puerperal de 127 vacas de corte e leite das 353 fêmeas utilizadas no experimento de Silva et al. (2001).

$\mathrm{Na}$ busca de identificar um produto comercial que melhore a taxa de prenhez dos protocolos de inseminação em tempo fixo (IATF), a homeopatização de compostos de fácil administração a um rebanho é um dos pontos de novas pesquisas. Gonçalves et al. (2012) trabalharam com embriofixador ${ }^{\circledR}$ homeopatizado, tratando as fêmeas ( $50 \mathrm{~g} / \mathrm{animal} / \mathrm{dia}$ junto a um concentrado) por um período de 30 dias após a inseminação. Os resultados mostraram um aumento de $12 \%$ na taxa de prenhez das fêmeas do grupo homeopatizado, quando comparado ao grupo controle que consumiu apenas concentrado.

$\mathrm{Na}$ tentativa de melhorar os resultados produtivos de sete doadoras de ovócitos da raça Nelore Costa Filho et al. (2013) avaliaram a produção e a qualidade ovocitária e embrionária das fêmeas, além da taxa de prenhez das receptoras inovuladas com os embriões produzidos. O complexo homeopático fornecido (10 gramas/animal/dia) era composto por Pulsatilla nigricans $12 \mathrm{CH}$, Apis mellifera $6 \mathrm{CH}$ e $\mathrm{CaCO}_{3}($ Carbonato de Cálcio). Todas as matrizes consumiram o produto por 20 dias antes de cada aspiração, porém o composto homeopático estudado não melhorou a fertilidade. No mesmo sentido do estudo, porém, como o objetivo de verificar melhora na taxa de prenhez das receptoras de embrião (Bos taurus $x$ Bos indicus), o composto homeopático Seppia succus $15 \mathrm{CH}$, Sulphur $10 \mathrm{CH}$ e $\mathrm{CaCO}_{3}$ foi administrado a 80 fêmeas em duas estações do ano (seca e chuvosa), mas os resultados não mostraram diferença significativa na taxa de prenhez em relação ao grupo tratado e o controle em nenhuma das duas estações (Souza et al., 2013).

Lima (2011) utilizou FSH dinamizado (dFSH) no cultivo in vitro de folículos pré antrais de ovinos inclusos em fragmentos de tecido ovariano, e encontrou que, na $6 \mathrm{CH}$, 
com o dFSH adicionado a cada 24 horas após o início do cultivo, houve crescimento folicular após sete dias de cultivo, mantendo a integridade ultraestrutural e a viabilidade dos folículos.

Conduzindo um experimento em uma granja de suínos por dois anos, Soto et al. (2008) observaram diferença significativa nos resultados dos tratamentos homeopáticos (H) e alopáticos (A). A eficiência reprodutiva foi medida por diversos parâmetros que favoreceram o tratamento homeopático: repetição de cio $(13,7 \% \mathrm{H}$ vs 30,1\% A), leitões nascidos vivos $(90,1 \% \mathrm{H}$ vs $87,1 \% \mathrm{~A})$, natimortos $(9,6 \% \mathrm{H}$ vs $12,3 \%$ A) e taxa de mortalidade na maternidade $(14,9 \% \mathrm{H} v s \quad 19,4$ $\%$ A). De forma similar Vuaden (2005) obteve aumento significativo no número de partos e taxa de nascimento após tratamento de porcas com produtos homeopáticos.

A utilização de princípios homeopáticos já vem sendo testada na reprodução de machos (LOBREIRO, 2007; SOUZA et al., 2012), demonstrando que podem trazer respostas positivas. A utilização de tratamentos alternativos em animais dos quais se processa material biológico como sêmen, pode ser extremamente interessante principalmente no sentido de contornar os efeitos negativos que o manejo intensivo de um Centro de Coleta e Processamento de Sêmen (CCPS) pode acarretar no perfil seminal ou para melhorar a congelabilidade, característica esta não necessariamente vinculada à capacidade reprodutiva intrínseca do indivíduo. Souza et al. (2012), utilizando diversos princípios homeopáticos conseguiram melhorar a eficiência de coleta e congelabilidade do sêmen de bovinos mantidos em CCPS. A Avena sativa é um medicamento homeopático utilizado em dinamizações mais baixas que, adicionado aos diluentes para resfriamento de sêmen suíno, melhorou a motilidade e vigor até 48 e 72 horas pós-coleta (SOTO et al., 2009).

Lobreiro (2007) tratou com Pulsatilla nigricans um touro da raça Nelore que apresentava infertilidade à três anos. Após o tratamento a qualidade seminal foi aumentada, diminuindo os defeitos espermáticos totais, aumentando a motilidade e volume do ejaculado. Entretanto, com apenas um animal observado, não é possível concluir que a mudança observada foi devida somente a esse tratamento.

Soto et al. (2010) realizaram a inseminação artificial em fêmeas bovinas com sêmen tratado com Avena sativa $(6 \mathrm{CH})$, e encontraram melhora significativa no número de partos, com menos matrizes retornando ao estro após a inseminação. Este estudo demonstra um grande impacto econômico na produção do rebanho, melhorando a taxa de nascimento e diminuindo custos

No desenvolvimento de projetos de pesquisa com a utilização de princípios homeopáticos, tentativas devem ser realizadas, para que a substância dinamizada em teste possa ser adaptada ao desenho do estudo proposto, pois as informações devem ser ajustadas de acordo com a variação de cada espécie (TEIXEIRA, 2006). O autor pondera que deve-se considerar os seguintes parâmetros: a potência do medicamento, tempo de tratamento e tempo de resposta.

\section{Considerações Finais}

Os tratamentos existentes mesmo que para os animais são baseados no Tratado de Medicina Humana, porém, os homeopatas utilizam do bom senso na transferência de informações para cada espécie, realizando um tratamento espécie-específico. Dessa forma, novos estudos devem ser desenvolvidos. $\mathrm{O}$ uso da homeopatia na reprodução se faz importante na busca de melhorias da fertilidade animal, tanto no diz respeito ao tratamento de patologias quanto à eficiência reprodutiva.

\section{Referências}

AMARAL, M. T. C. G. Homeopatia veterinária: estratégias de ação. International Journal of High Dilution

Research, São Paulo, v.1, n.1, p. 8-10, 2002.

ARENALES, M. D. C. Homeopatia em gado de corte. CONFERÊNCIA VIRTUAL GLOBAL SOBRE PRODUÇÃO ORGÂNICA DE BOVINOS DE CORTE, 1., 2002, Concórdia. Anais... Concórdia: UNC. 2002. 11 p.

BENITES, N. R. Homeopatia. In: SPINOSA, H. S.; GORNIAK, S. L.; BERNARDI, M. M. Farmacologia aplicada a medicina veterinária. 4. ed. Rio de Janeiro: Guanabara Koogan, 2006, p. 827-835.

BRASIL. Conselho Federal de Medicina Veterinária e Zootecnia- CFMV. Resolução no ${ }^{625}$, 16/03/1995, Regulamenta o registro de título de especialidades (homeopatia), Brasília, DF, 1995.

CASTILHOS, L. R. et al. Avaliação da terapêutica homeopática nas ovariopatias císticas de bovinos leiteiros. Homeopatia Brasileira, Rio de Janeiro, v. 9, n. 1, p. 5-15, 2003.

CESAR, A. T. Dinamização. Cultura homeopática, São Paulo, v. 2, n. 5, p. 15-41, 2003.

CHABEL, J. C. et al. Efeito de um complexo homeopático "Homeobase Convert $\mathrm{H}^{\circledR}$ em ovinos sob condição de restrição alimentar. Brazilian Journal of Veterinary Research and Animal Science, São Paulo, v. 46, n. 5, p. 412-423, 2009.

COSTA, N. C.; ARAÚJO, R. L.; FREITAS, G. B. L. Homeopatia: um campo terapêutico fundamental no cuidado veterinário de animais de produção. Revista SaulosGuarapuava, v. 3, n. 2, p. 75-89, 2010.

COSTA FILHO, L. C. C. et al. Produção ovocitária e embrionária de doadoras da raça Nelore tratadas com um complexo homeopático. In: CONGRESSO BRASILEIRO DE REPRODUÇÃO ANIMAL, 20., 2013, Uberlândia. Anais... Uberlândia: O Congresso, 2013. CD-ROM.

EIZAYAGA, F. X. Tratado de medicina homeopática, Buenos Aires: Marecel, 1992. 399 p.

FERREIRA, L. C. Avaliação da qualidade de carcaça e da composição corporal de dois grupos genéticos, tratados ou não, com uma mistura de "calcárea carbônica" 30CH e "calcarea phosphorica" 30CH. 2005. 22 f. Monografia (Especialização em Zootecnia) - 
Universidade Estadual de Mato Grosso do Sul, Aquidauana, 2005 .

GONÇALVES, D. K. Efeito do produto homeopático embriofixador $^{\circledR}$ sobre a taxa de prenhez em novilhas de corte. In: SALÃO INTENACIONAL DE ENSINO, PESQUISA E EXTENSÃO, 4., 2012, Uruguaiana. Anais... Uruguaiana: Unipampa, IV Salão Internacional de Ensino, Pesquisa e Extensão. 2012.

KENT, J. T. Filosofia homeopática, Curitiba: Nova Época, 1993. $248 \mathrm{p}$

KUMAR, H. et al. Management of postpartum anoestrus in dairy animals with a homeopathic combination remedy. Indian Journalof Animal Science, New Delhi, v. 74, p. 739-740, 2004

LIMA, L. F. Utilização do hormônio folículo estimulante (FSH) homeopático no cultivo in vitro de folículos pré-antrais ovinos inclusos em fragmentos de tecido ovariano. 2011. 119 f. Dissertação (Mestrado em Ciências Veterinárias) - Universidade Estadual do Ceará, Fortaleza, 2011.

LOBREIRO, J. Homeopathic treatment for infertility in a prize Nelorebull. Homeopathy, Edinburgh, v. 96, p. 49-51, 2007.

LOPES, E. G. Homeopatia aplicada à parasitologia veterinária. In: CONGRESSO BRASILEIRO DE PARASITOLOGIA VETERINÁRIA \& SIMPÓSIO LATINO AMERICANO DE RICKETSIOSES, 12., 2004, Ouro Preto. Anais... Ouro Preto: Colégio Brasileiro de Parasitologia Veterinária. 2004.

LOPES, H. C.; ONSELEN, V. J. V.; SOUZA, A. S. Homeopatia no comportamento de camundongos sob estresse agudo. Revista Brasileira de Saúde e Produção Animal, Salvador, v.10, n. 4, p. 840-851, 2009.

MACEDO, N.; REAL, C. M. Homeopatia populacional na melhoria da fertilidade ovina em campo nativo, no Rio Grande do Sul. 2008. Disponível em: <http:// www.produzamelhor.com.br/publicacoestecnicas. php?menu $=$ artigos \& codigo $=13>$. Acesso em: 10 ago. 2012

MARTINS, C. R. et al. Tratamento de mastite subclínica por meio de suplementação mineral homeopática da dieta de vacas leiteiras em lactação - Estudo de Caso. Cultura Homeopática, n.19, p. 16-19, 2007.

MITIDIEIRO, A. M. A. Potencial do uso de homeopatia, bioterápicos e fitoterapia como opção na bovinocultura de leite: avaliação dos aspectos sanitários e de produção. Florianópolis, 2002. 119 f. Dissertação (Mestrado em Agrossistemas) - Universidade Federal de Santa Catarina, Florianópolis, 2002.
MONCAYO, F. Efficacy of homeopathic preparations of autogenous mastitis causing organisms in the prevention of mastitis in dairy cattle. Organic Farming Research Foundation Project Report, v. 99, n. 3, p.1-15, 2000.

PIRES, M. F. A, A homeopatia para os animais. Juiz de Fora: Comunicado Técnico 46 - Embrapa Gado de Leite, $2005.4 \mathrm{p}$

RAJKUMAR, R. et al. Effect of a homeopathic complex on oestrus induction and hormonal profile in anoestrus cows. Homeopathy, Edinburgh, v. 95, p.131-135, 2006.

SILVA, C. T. L.; MCMANNUS, C.; RUNPE, R. Efeito da Pulsatilla nigricans aplicada em um ponto de acupuntura na redução do puerpério bovino. Homeopatia Brasileira, Rio de Janeiro, v. 7, n.1, p.13-21, 2001.

SILVA, J. R. M. et al. Suplementação de vacas leiteiras com homeopatia: células somáticas do leite, cortisol e imunidade. Arquivo Brasileiro de Medicina Veterinária e Zootecnia, Belo Horizonte, v. 63, n. 4, p. 805-813, 2011.

SOARES FILHO, V. C.; CAETANO, H. Desempenho de bovinos de corte suplementados com cr-levedura e produto homeopático. Boletim de Indústria Animal, Nova Odessa, v. 59, n. 2, p.177-184, 2002.

SOTO, F. R. M. et al. Avaliação dos índices zootécnicos de uma granja comercial de suínos com a utilização do tratamento homeopático. Veterinária e Zootecnia, Botucatu, v. 15, n. 3, p. 577-586, 2008.

SOTO, F. R. M. et al. Efeito da Avena Sativa CH6 no metabolismo do sêmen diluído de suínos. Veterinária e Zootecnia, Botucatu, v.16, n. 2, p. 367-72, 2009.

SOTO, F. R. M. et al. Reproductive performance of sows inseminated with diluted semen treated with homeopathic medicine. International Journal of High Dilution Research, Guaratingueta, v. 9, n. 30, p. 51-57, 2010.

SOUZA, C. C. et al. Homeopatia e eficiência reprodutiva de receptoras de embrião bovino. In: CONGRESSO BRASILEIRO DE REPRODUÇÃO ANIMAL, 20., 2013, Uberlândia. Anais... Uberlândia: O Congresso, 2013. CDROM.

SOUZA, M. F. A. Homeopatia veterinária. 2002. Disponível em: < http://canildw.com.br/tecnica/ medicinaalternativa/HOMEOPATIA\%20VETERINARIA. pdf $>$. Acesso em: 10 jun. 2012.

SOUZA, M. F. A. et al.The effect of homeopathy on the fertility of bulls with previous reproductive disorders. Homeopathy, Edinburgh, v. 101, p. 243-245, 2012.

TEIXEIRA, M. A. et al. Effect of a bio stimulatory homeopathic complex in mice subjected to feed stress. In: INTERNATIONAL CONGRESS IN THE FUTURE OF ANIMAL RESEARCH, 1., Rio de Janeiro. Anais... Rio de 
Janeiro: [s.n.], p. 56, 2003.

TEIXEIRA, M. Z. Homeopatia: ciência, filosofia e arte da cura. Revista de Medicina, São Paulo, v. 85, n. 2, p.30-43, 2006.

VARSHENEY, J. P.; NARESH, R. Comparative efficacy of homeopathie and allopathic systems of medicine in the managements of Indian dairy cows. Homeopathy, Edinburgh, v. 94, p. 81-85, 2005.

VUADEN, E. R. Homeopatia na suinocultura. 2005. 97 f. Monografia (Especialização e Homeopatia Veterinária) - Faculdade de Ciências da Saúde de São Paulo, Centro de Ensino Superior de Homeopatia. São Paulo, 2005.

ZORZATTO, C.; TEIXEIRA, M. A.; CARVALHO, T. B. S. Efeito do Convert $\mathrm{H} \circledast$-Complexo homeopático bioestimulatório - em gerações de camundongos (Mus musculus). Revista da Universidade Rural, Série e

Ciência da Vida, Seropédica, v. 25, p. 7-9, 2005.

Recebido em 17/06/2013 Aceito em 06/06/2014 